\title{
Oxidative stress induced by Se-deficient high-energy diet implicates neutrophil dysfunction via Nrf2 pathway suppression in swine
}

\author{
Tianshu Yang ${ }^{1, *}$, Zeping Zhao ${ }^{2, *}$, Tianqi Liu ${ }^{1}$, Ziwei Zhang ${ }^{1}$, Pengzu Wang ${ }^{1}$, Shiwen $\mathrm{Xu}^{1,3}$, \\ Xin Gen Lei ${ }^{2}$, Anshan Shan ${ }^{1}$ \\ ${ }^{1}$ Northeast Agricultural University, Harbin, P. R. China \\ ${ }^{2}$ Department of Animal Science, Cornell University, Ithaca, NY, USA \\ ${ }^{3}$ Key Laboratory of Animal Cellular and Genetic Engineering of Heilongjiang Province, Northeast Agricultural University, \\ Harbin, China \\ *These authors are contributed equally to this work
}

Correspondence to: Shiwen Xu, email: shiwenxu@neau.edu.cn

Xin Gen Lei, email: xI20@cornell.edu

Anshan Shan, email: asshan@neau.edu.cn

Keywords: high-energy, se deficiency, neutrophils, oxidative stress, Nrf2

Received: November 09, $2016 \quad$ Accepted: December 27, 2016

Published: January 07, 2017

\section{ABSTRACT}

The mechanism of the interaction between Se deficiency and high energy remains limited. The aim of the current study was to identify whether Se-deficient, high-energy diet can induce oxidative stress, and downregulate the Nrf2 pathway and phagocytic dysfunction of neutrophils. We detected the phagocytic activity, ROS production, protein levels of Nrf2 and Nrf2 downstream target genes, and the mRNA levels of 25 selenoproteins, heat shock proteins, and cytokines in neutrophils. Cytokine ELISA kits were used to measure the serum cytokines. The concentration of ROS was elevated $(P<0.05)$ in obese swine fed on a low Se diet (less than $0.03 \mathrm{mg} / \mathrm{kg} \mathrm{Se}$ ) compared to control swine. The protein levels of $\mathrm{Nrf2}$ and its downstream target genes were depressed during Se deficiency and high-energy intake. The mRNA levels of 16 selenoproteins were significantly decreased $(P<0.05)$ in the Se-deficient group and Se-deficient, high-energy group compared to the control group. However, the mRNA levels of 13 selenoproteins in peripheral blood neutrophils were upregulated in high energy group, except TrxR1, SelI and SepW. In summary, these data indicated that a Se-deficient, high-energy diet inhibits the Nrf2 pathway and its regulation of oxidative stress, and prompted a pleiotropic mechanism that suppresses phagocytosis.

\section{INTRODUCTION}

The nutrient elements of animal diets are key to redox homeostasis and the functions of the body's immune system. When there is redox equilibrium dysfunction, the functions of the immune system are impaired as a result of the selenium and lipid-stimulated oxidants and antioxidants [1-3]. Several studies reported that Se deficiency disrupted the expression of selenoproteins in many tissues and organs of livestock and humans $[4,5]$. In these situations, immune system dysfunction is caused by oxidative stress and the generation of ROS. Selenoproteins, such as GPX, TRXR, SEPP1, SelN and SelW, exert antioxidative ability [6]. High-energy diet results in obesity, the continuous generation of ROS from the stimulation of a metabolic surplus, and a drop in the immune function of the body by remaining in a persistent condition of oxidative stress. This induces low-grade, chronic inflammation from the generation of lipid peroxidation and enhances the expression quantity of pro-inflammatory cytokines, such as IL-1, IL-6, IL-10, TNF- $\alpha$, and COX-2 [7, 8].

The nature of oxidative stress underscoring the production of ROS can be induced by external factors. In homeostatic conditions, Nrf2, which has no functional activity, exists in the cytoplasm and combines with Keap1. In conditions of oxidative stress, Nrf2 dissociates from Keap1, moves to the nucleus, and combines with ARE to form Nrf2-ARE, activating downstream extranuclear genes such as sod2, gpxl, trxRl, ho-1 and catalase [9]. 
The Nrf2 pathway has been shown to have both up- and down-regulated functions [10]. Nrf2 pathway could also suppress potent phagocytic activity [11, 12]. In studies in the bone marrow, the expression of glutathione in immature dendritic cells decreased with damaged phagocytosis and without Nrf2 in the bone marrow [13]. The Nrf2 pathway can be adjusted by many factors, such as the intake of Se and energy. Nrf2 will stimulate the expression of up and downstream genes through relevant metabolic pathways [14]. At present, as shown in human and mouse experiments, Se deficiency activated the Nrf2 pathway. This increased the expression of Nrf2 so that it could increase the expression of relevant downstream antioxidant genes, including selenoproteins GPX1 and TRXR-1 [15]. In relevant experiments looking at high-fat intake, fatty liver induced by high-fat diet suppressed the Nrf2 pathway, whose main inhibition mechanism might be associated with a chronic pathological process [16]. The previous research also indicated that the inhibition factors about Nrf2 pathway also included high blood pressure, high-glucose, premature aging and zinc deficiency [17-19]. When the Nrf2 pathway was restrained, the body's antioxidant ability decreased, leading to the promotion of oxidative stress and the dysfunction of the immune system.

Invading pathogens can be killed by neutrophils through phagocytosis and the release of inflammatory factors (IL-1 $\beta$, IL-6, IL-8, COX-2) [20]. Neutrophil activation generates large quantities of ROS to activate the Nrf2 pathway and, as a result, the function of neutrophils is feedback inhibited $[21,22]$. Related research in mice fed with high-fat diets and with a knockout in ApoE found that inflammatory monocytes and neutrophils could be regulated by nestin $(+) \mathrm{BM}$ cells, indicating that these cells could regulate inflammatory cells during chronic inflammation [23]. Similar results were observed in wild-type mice fed high-fat diets. Monocyte-depletion analyses were performed, showing that neutrophils were preferentially recruited to the femoral artery, and proved that PMN-derived MCP-1 was important in the accumulation of leukocytes [24]. The dysfunction of neutrophils is associated with a Se-deficient diet. However, the effect of Se-deficiency and a high-energy diet on neutrophil dysfunction is still not clear. We hypothesized that Se-deficiency and high-energy diets result in a phagocytosis problem in neutrophils by restraining the Nrf2 pathway from producing persistent oxidative stress, resulting in the further dysfunction of the immune system. Herein, the influence and mechanism on neutrophils associated with a Se-deficient, high-energy diet can be clarified by monitoring phagocytosis activity, and the concentration of reactive oxygen. To further link ROS generation to neutrophil phagocytosis, we evaluated the level of Nrf2, the downstream genes, 25 selenoproteins, HSPs and relevant cytokines, which were acquired from swine fed with a Se-deficient, high-energy diet.

\section{RESULTS}

\section{Se-deficient high-energy diet inhibited neutrophil phagocytosis}

To confirm whether Se deficiency and high-energy diets affect the function of neutrophils, swine neutrophils were freshly isolated from a control group, $\mathrm{Se}^{-}$group, energy $^{+}$group, and $\mathrm{Se}^{-}$-energy ${ }^{+}$group. As expected, the Se-deficient and high-energy treatment prohibited the ability of neutrophils to endure phagocytosis compared with the control group (Table 1). Compared to the control group, dietary Se deficiency significantly decreased the neutrophils phagocytic activity $(P<0.05)$ as well as the OD phagocytic activity index, which was decreased $(P<0.05)$. Supplementation of energy resulted in a decreased percentage of phagocytosis compared with the control group, and no significant differences between the energy ${ }^{+}$group and $\mathrm{Se}^{-}$group were observed in these parameters. These data demonstrate a critical role for $\mathrm{Se}^{-}$and energy ${ }^{+}$in regulating neutrophil phagocytosis.

\section{Se-deficient high-energy diet promoted the generation of ROS in neutrophils}

To assess the reason why neutrophil phagocytosis is decreased by Se and energy status, we evaluated the degree of oxidative stress by detecting the production of ROS in swine neutrophils. Figure 1A shows typical images of ROS under various conditions. As shown in Figure 1B, a Se-deficient, high-energy diet significantly increased ROS production $447 \pm 23.56$-fold compared with the control group. Treatment with a Se-deficient and high-energy diet showed a similar, strengthened effect on ROS production, induced from $100 \pm 5.64$-fold over the control to $375 \pm 17.81$-fold and from $100 \pm 5.64$-fold over the control to $217 \pm 16.73$-fold, respectively.

Furthermore, we determined whether there was a change in the heat shock protein (HSP) gene expression in neutrophils. The mRNA levels of Hsp40, Hsp60, Hsp70 and Hsp90 were significantly increased in the low Se and high-energy groups $(P<0.05)$ compared with the corresponding control group (Figure 1C). Specifically, the mRNA levels of Hsp60, Hsp70 and Hsp90 increased $\sim 2000 \%$ due to the high-energy and low Se diet feeding. HSPs showed the highest expression level among all the groups. Notably, the lowest range of Hsp40 mRNA expression during the Se-deficient and high-energy condition was observed.

\section{Se-deficient high-energy diet influenced the mRNA expression of selenoproteins}

We detected the mRNA expression of 25 selenoproteins. However, the remaining 9 selenoproteins were expressed faintly in neutrophils. As shown in Figure 2, 
Table 1: Mean values within a row with different letters are significantly different $(P<0.05)$

\begin{tabular}{|c|c|c|c|c|}
\hline & CD & SDD & HED & SED \\
\hline Phagocytic activity $(\%)^{1}$ & $64.87 \pm 0.287^{C}$ & $49.54 \pm 0.339^{A a}$ & $55.46 \pm 0.462^{\mathrm{Bb}}$ & $43.77 \pm 0.138^{\mathrm{Ab}}$ \\
\hline Index of phagocytic activity ${ }^{2}$ & $1.47 \pm 0.119^{C}$ & $1.24 \pm 0.213^{\mathrm{Aa}}$ & $1.26 \pm 0.193^{\mathrm{Aa}}$ & $1.16 \pm 0.088^{\mathrm{Bb}}$ \\
\hline
\end{tabular}

${ }^{1}$ Number of white cells containing at least three engulfed particles per 100 white cells (neutrophils).

${ }^{2}$ Number of engulfed particles per total number of neutrophils observed.

the mRNA abundances of the selenoproteins (TRXR-1, GPX1, GPX2, GPX3, GPX4, SeP, SelH, SEPHS, SelI, SelM, SEPP1, SelT, SelX, SelK, SepN, SepW) were significantly decreased $(P<0.05)$ in the $\mathrm{Se}^{-}$group and $\mathrm{Se}^{-}$-energy ${ }^{+}$group compared to the control group in swine peripheral blood neutrophils. However, surprisingly, the levels of 13 selenoproteins were regulated in an opposite fashion in the energy ${ }^{+}$group, except TRXR-1, SelI and SepW. Dramatically, the results showed that the deficiency of dietary Se and high-energy intake have the opposite effect on regulating selenoprotein profiles in neutrophils. Interestingly, the GPX family levels were more sensitive to the $\mathrm{Se}$ and energy status amongst the $\mathrm{Se}^{-}$group, the energy ${ }^{+}$group and $\mathrm{Se}^{-}$-energy ${ }^{+}$group. Obese pigs with the low Se diet had neutrophil concentrations of GPX1, GPX2, GPX3 and GPX4 that were 38\%, 28\%, 31\% and $18 \%$ lower $(P<0.05)$ than the control group, respectively. Thus, the range of the mRNA expression of selenoproteins in the control group and Se- group are similar to energy ${ }^{+}$ group and $\mathrm{Se}^{-}$-energy ${ }^{+}$group.
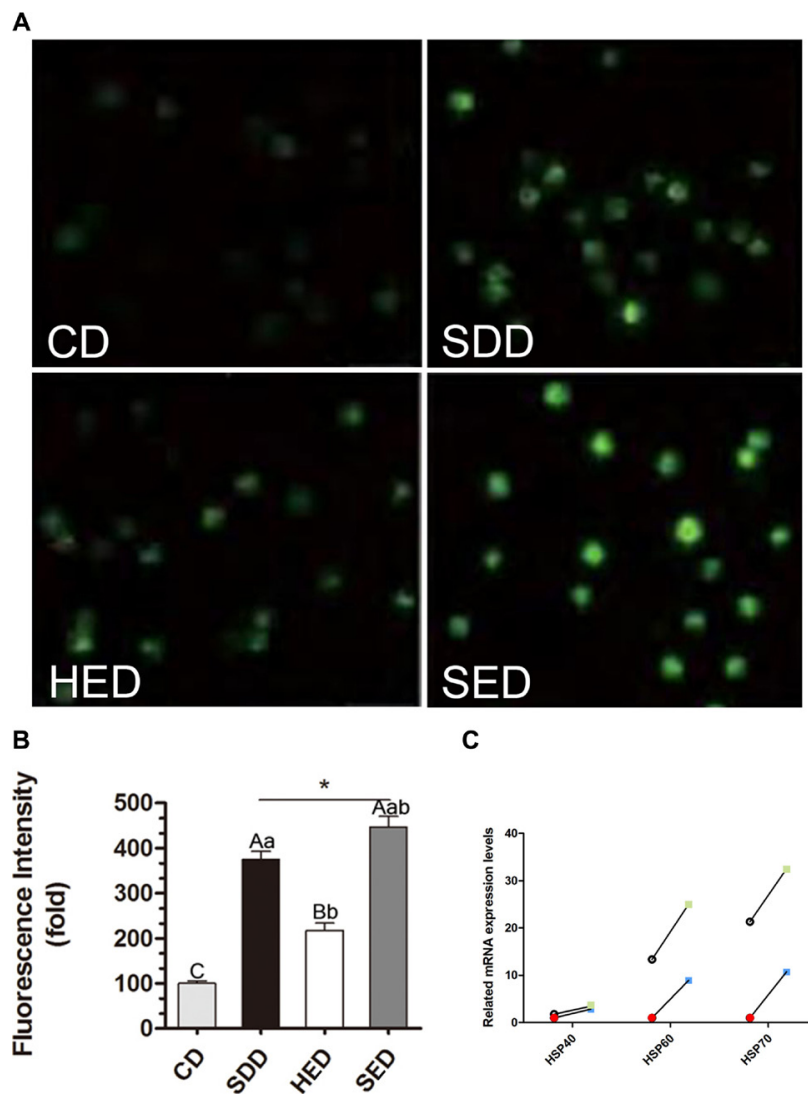

C

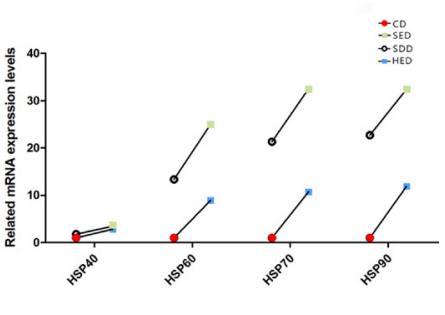

Figure 1: Se-deficient diet, high-fat diet and Se-deficient, high-energy diet induced ROS levels in neutrophils. (A) Immunofluorescence analysis was performed using DCFH-DA (green fluorescence, $5 \mathrm{mM}$ ). Neutrophils were visualized using fluorescence microscopy. (B) Three different fields were randomly counted for green positive neutrophils using Image J and the average fluorescence intensity of cells in eac diet were compared to that of control. Statistical significance was calculated by one-way ANOVA test, where bars that are significantly different $(p<0.05)$ from each other do not share the same letter. The data were expressed as the mean \pm SD $(n=10)$. (C) Neutrophils mRNA levels of Hsp40, Hsp60, Hsp70 and Hsp90 in different treated groups. Bars that do not share the same letters are significantly different $(p<0.05)$ from each other. The data were expressed as the mean $\pm \operatorname{SD}(n=10)$. 


\section{Se-deficient high-energy diet downregulated Nrf2 and its downstream target genes}

To determine whether the Nrf2 pathway regulated oxidative stress under a situation of Se deficiency and high-energy, we evaluated the protein level of Nrf2 and the main downstream target genes (SOD2, HO-1, Gpx1, TrxR1, catalase) in the Nrf2 pathway. Protein levels of Nrf2 were decreased in the Se deficiency, high-energy and Se-deficient, high-energy treatment compared to the control group (Figure 3). As expected, the differential expression of five down-regulated genes (SOD2, HO-1, Gpx1, TrxR1, and catalase) was observed in the Se-group and $\mathrm{Se}^{-}$-energy ${ }^{+}$group. These data indicate that $\mathrm{Se}^{-}$and $\mathrm{Se}^{-}$-energy ${ }^{+}$treatment downregulated $\mathrm{Nrf} 2$ and $\mathrm{Nrf} 2$ downstream target genes. However, protein expression of the three down-regulated genes (SOD2, Gpx1, and TrxR1) and two up-regulated genes (HO-1, catalase) was examined in the energy ${ }^{+}$group. Taken together, these data show that Se and energy status reduced protein level of Nrf2 and inhibit downstream proteins of the Nrf2 pathway compared with the control group.

\section{Se-deficient high-energy diet changed the expression of cytokines in serum}

To evaluate whether a Se-deficient, high-energy diet induced inflammation response in the body, we examined the level of cytokines in serum by ELISA. As shown in Figure 4, the level of IL- $1 \beta$ and TNF- $\alpha$ in the serum elevated $\sim 3.0$ and $\sim 0.3$-fold, respectively, with $\mathrm{Se}^{-}$, energy ${ }^{+}$and $\mathrm{Se}^{-}-$energy ${ }^{+}$treatment, which are consistent with the mRNA data in the neutrophils. However, the level of IFN- $\gamma$ and IL-2 significantly decreased $(P<0.05)$ in the $\mathrm{Se}^{-}$, energy ${ }^{+}$, and $\mathrm{Se}^{-}$-energy ${ }^{+}$groups compared to the control group. The expression of IL-10 was reduced by Se deficiency and Se-deficient, high-energy treatment, but the energy ${ }^{+}$group significantly increased its level from $1.638 \pm 0.109$ to $1.786 \pm 0.025$-fold.

\section{Se-deficient high-energy diet upregulated mRNA expression of inflammatory factors}

The mRNA levels of IL- $1 \alpha$, IL-1 $\beta$, IL-6, IL-8, IL9, Nf-kbp50, Nf-kbp65, TNF- $\alpha$, INOS, and COX-2 were elevated in the neutrophils of the $\mathrm{Se}^{-}$, energy ${ }^{+}$, and $\mathrm{Se}^{-}-$ energy $^{+}$groups compared with the corresponding control group (Figure 5). In the $\mathrm{Se}^{-}$-energy ${ }^{+}$group, the mRNA expression level of IL-1 $\beta$, IL-6 and IL-8 were $128 \%$, $405 \%$ and $313 \%(P<0.05)$ greater than $\mathrm{Se}^{-}$group, respectively. The rest of the genes showed an opposite, downward fashion, with the obvious decline in IFN- $\gamma$ and TGF- $\beta 1$. Inflammation-related gene expression was highly stimulated in the high-energy and low Se feeding group. Of these, IL- $1 \alpha$, iNOS and COX-2 showed the highest responsiveness in neutrophils of the Se deficiency and high-energy condition.

\section{DISCUSSION}

Nutrient elements act in balance with oxidation metabolism and immune system function. For instance, Se deficiency leads to reduced selenoprotein content in tissues and organs $[4,5,25]$ and results in chronic inflammation, which is induced by oxidative stress [26], which in turn hampers immunity. These changes will also impair the functions of immune cells [27]. In addition, a high energy content diet would give rise to obesity and induce chronic oxidative stress, which would further bring about chronic inflammation [28] and disrupt the functioning of immune cells [29]. This study proved that a Se-deficient, high-energy diet can inhibit the Nrf2 pathway in neutrophils, trigger sustaining oxidative stress and reduce phagocytosis. Moreover, a Se-deficient,

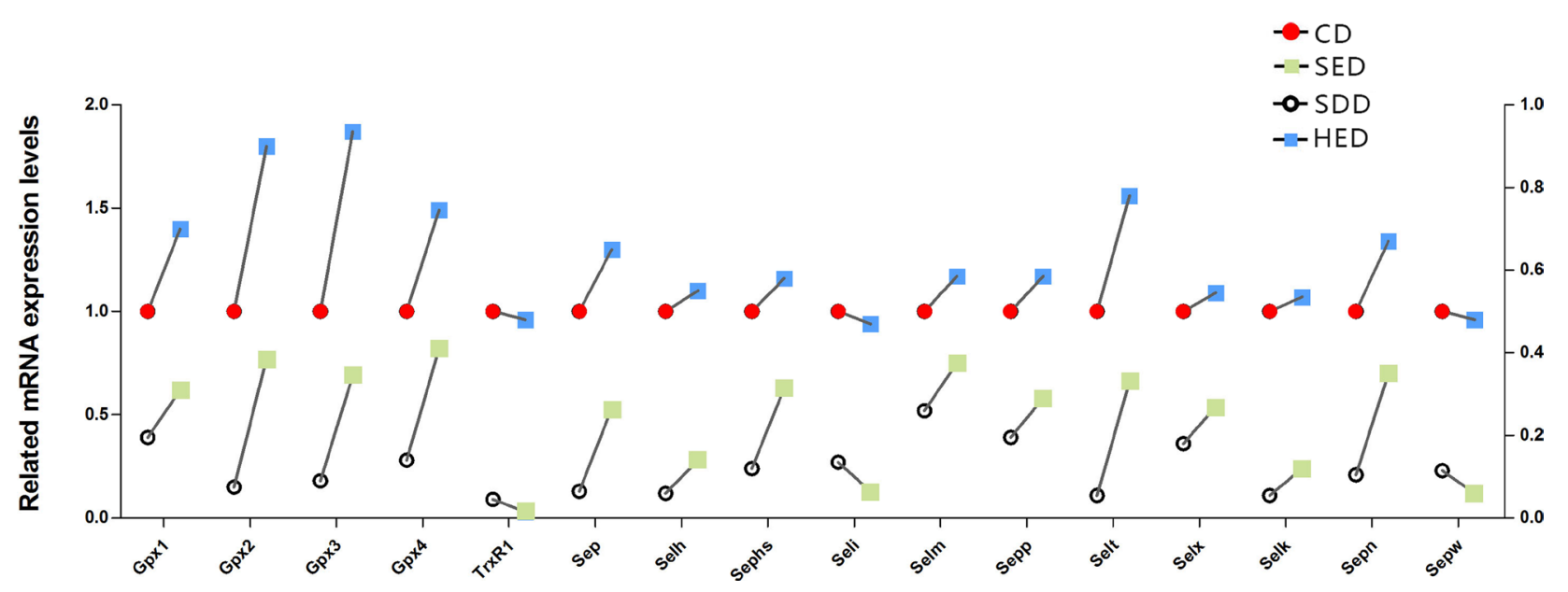

Figure 2: Neutrophils mRNA levels of selenoproteins genes (TrxR1, Gpx1, Gpx2, Gpx3, Gpx4, Sep, Selh, Sephs, Seli,Selm, Sepp1, Selt, Selx, Selk, Sepn and Sepw) in the different treated regions. Bars that do not share the same letters are significantly different $(p<0.05)$ from each other. The data were expressed as the mean $\pm \operatorname{SD}(n=10)$. 
high-energy daily ration can cause chronic inflammation in the body.

Many references indicated that the oxidative stress of tissues and organs induced by the Se-deficient diet might be associated with selenoproteins [30-33]. The rationale is that the reduced expression of antioxidative selenoproteins may lead to redox homeostasis disorder and ROS accumulation, which encourages oxidative stress [6]. Studies on neutrophils in Se-deficient broilers found that a shortage of selenium in daily rations lifted the level of Hsp40, Hsp60, Hsp70, Hsp90, iNOS and NO production by a large margin [34]. In our experiment, Se deficiency diet lowered the expression of 16 selenoproteins, but boosted Hsp40, Hsp60, Hsp70 and Hsp90. The level of ROS also increased, suggesting that Se-deficient diet was responsible for oxidative stress in the peripheral neutrophils of swine. Furthermore, the high-energy diet involved in this experiment improved the expression of 13 selenoproteins in neutrophils, TRXR-1, SelI, and SepW excluded, which was partly consistent with the findings of our previous research. The latter tested the level of selenoproteins in pigs on high-fat diets and discovered that excessive fats enhanced 12 selenoproteins, respectively, in six types of tissues. Additionally, the expression of selenoproteins in different types of tissues was reduced. Additionally, the expression of TRXR-1 in the pancreas, hypothalamus and pituitary dropped [35], indicating that exorbitant fats are tissue-specific for the expressions of selenoproteins. High-energy daily rations could cause obesity. Obesity likely generates increased blood lipids

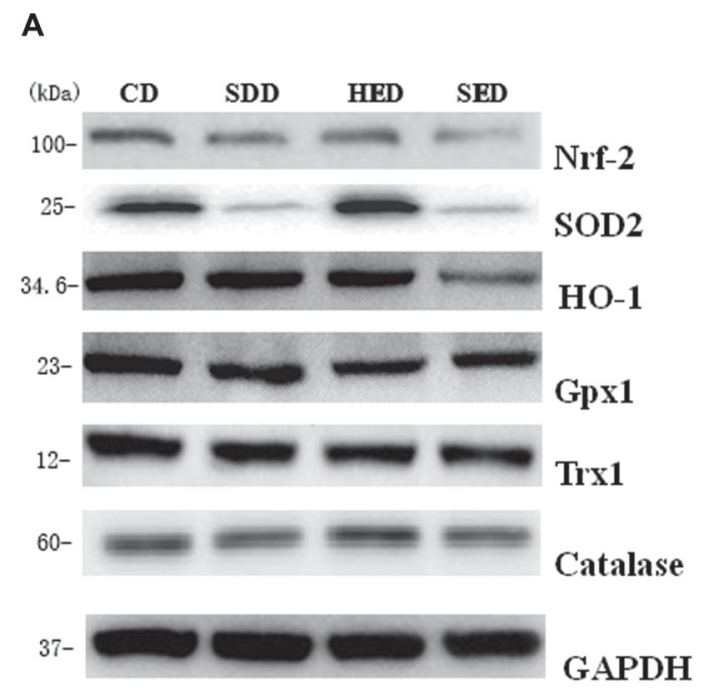

and ROS. The supposition that oxidative stress is attributed to high-energy diets has been confirmed by experiments on humans and a variety of animals [36-39]. In vitro experiments have demonstrated that fatty acids will bring about cellular oxidative stress [40-42]. In vitro cultured bovine hepatocytes applied with non-esterified fatty acids (NEFAs) hindered the expression of Nrf2 through the activation of ROS-p38-p53/Nrf2 signaling pathway, and, as a result, induced intracellular oxidative stress [43]. What's more, high-energy diets could impede the production of antioxidants and jeopardize the redox homeostasis by directing the Nrf2 signaling pathway to induce oxidative stress [44]. This experiment proved that a low level of Se and high content of energy prompts the production of ROS in neutrophils and triggers oxidative stress.

Some studies have verified that Nrf2 represents the center regulator of oxidative stress, which is normally located in the cytoplasm and dissociates with Keap1. In the case of cellular oxidative stress, once the system is activated, Nrf2 would enter the nucleus and bind with ARE to activate the downstream antioxidant genes of the Nrf2 pathway to restore the dynamic redox homeostasis [39]. The present experiment has shown that detected Nrf2 proteins were on the decline as the level of SOD2, GPX1, TRXR-1, HO-1, other downstream genes of the Nrf2 pathway and catalase proteins decreased. Because the expression of antioxidant genes was inhibited and substantial ROS could not be reduced, then sustained and unregulated oxidative stress was observed in neutrophils. Relevant studies demonstrated

B
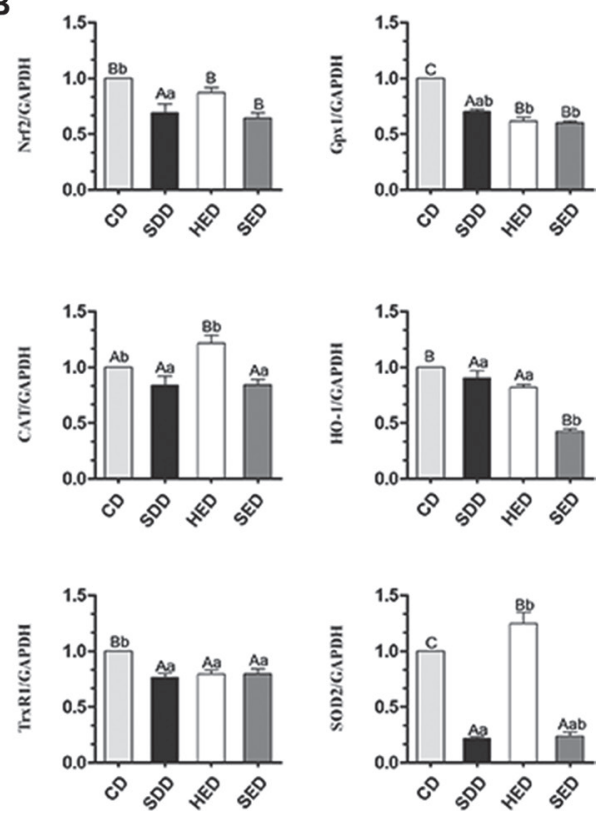

Figure 3: CD, SDD, HED and SED treatment for decreases in Nrf2-regulated antioxidant proteins in neutrophils. (A) Western blot analyses of Nrf2 regulated antioxidant proteins (Nrf2, TrxR1, HO-1, GPX1, catalase and SOD2) in SDD, HED and SED treated neutrophils. (B) Relative intensity of the protein signal was calculated using Image-J software and normalized to GAPDH. Statistical significance was calculated by a one way ANOVA test, where bars are significantly different $(p<0.05)$ from each other do not share the same letter. The data were expressed as the mean $\pm \operatorname{SD}(n=10)$. 
that many factors account for the inhibition of the Nrf2 pathway, such as chronic inflammatory diseases and hypertension controlled by obesity [16, 17]. Moreover, studies on Hutchinson-Gilford progeria syndrome (HGPS) found that the Nrf2 pathway participated in the oxidative stress induced by a high-fat diet [44]. That coincides with our results, which show that high-energy diet causes oxidative stress in neutrophils and obstructs the Nrf2 pathway. Studies on humans and mice taking in inadequate Se found that a low amount of Se activated the Nrf2 pathway and promoted the expression of the selenoprotein, TRXR-1, while reducing the amount of some selenoproteins in multiple organs [14, 45]. That was contrary to what was observed in this experiment. The conclusion that the expression of TRXR-1 improved disagreed with most relevant reports that Se deficiency
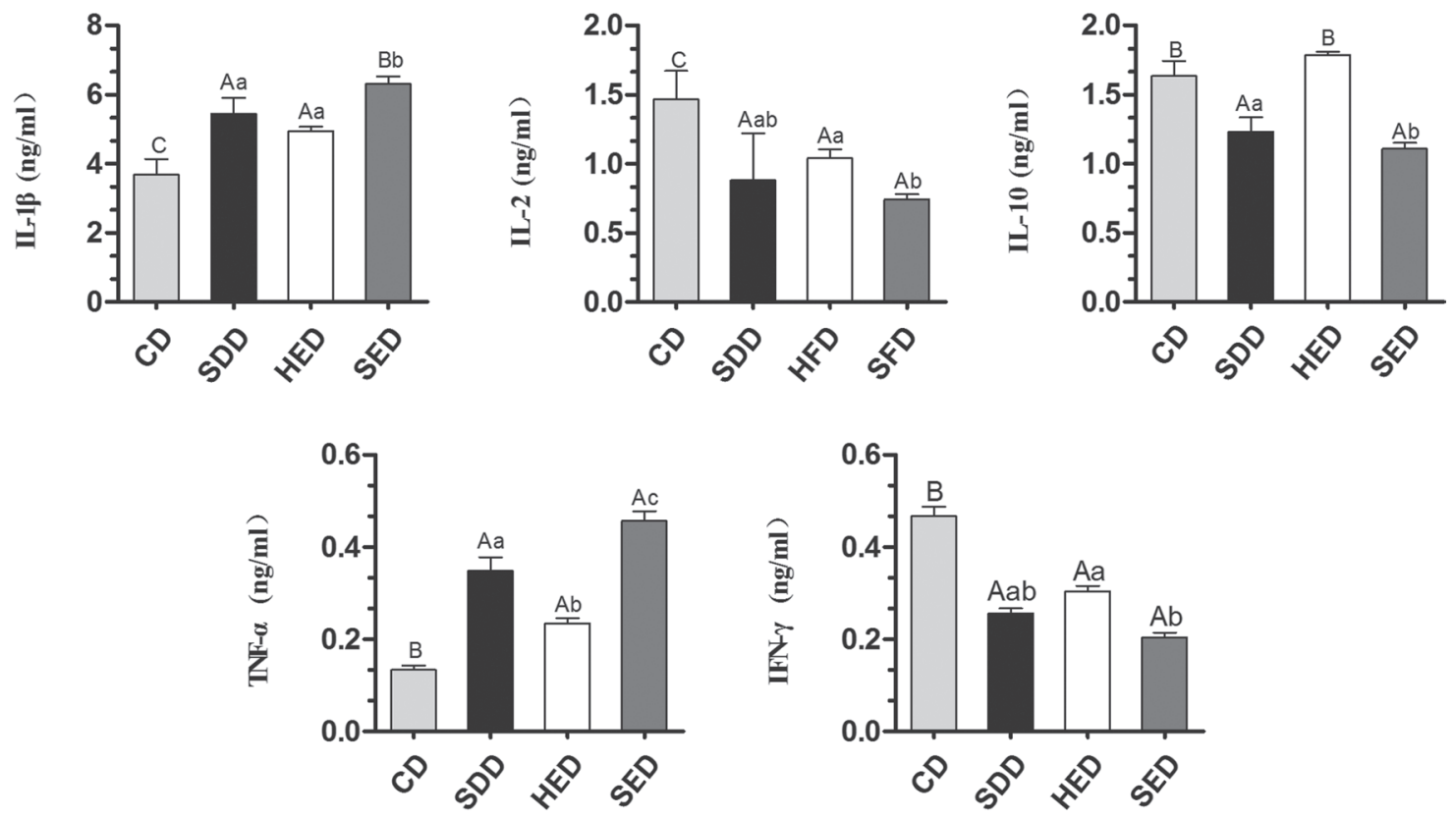

Figure 4: Different diets induce cytokines activation in serum. (A) Cytokines (IL-1 $\beta$, IL-2, IL-10, TNF- $\alpha$ and IFN- $\gamma$ ) in serum extracts was measured by ELISA. (B) Relative intensity of the cytokines levels was calculated using GraphPad Prism. Bars that do not share the same letters are significantly different $(p<0.05)$ from each other. The data were expressed as the mean $\pm \operatorname{SD}(n=10)$.

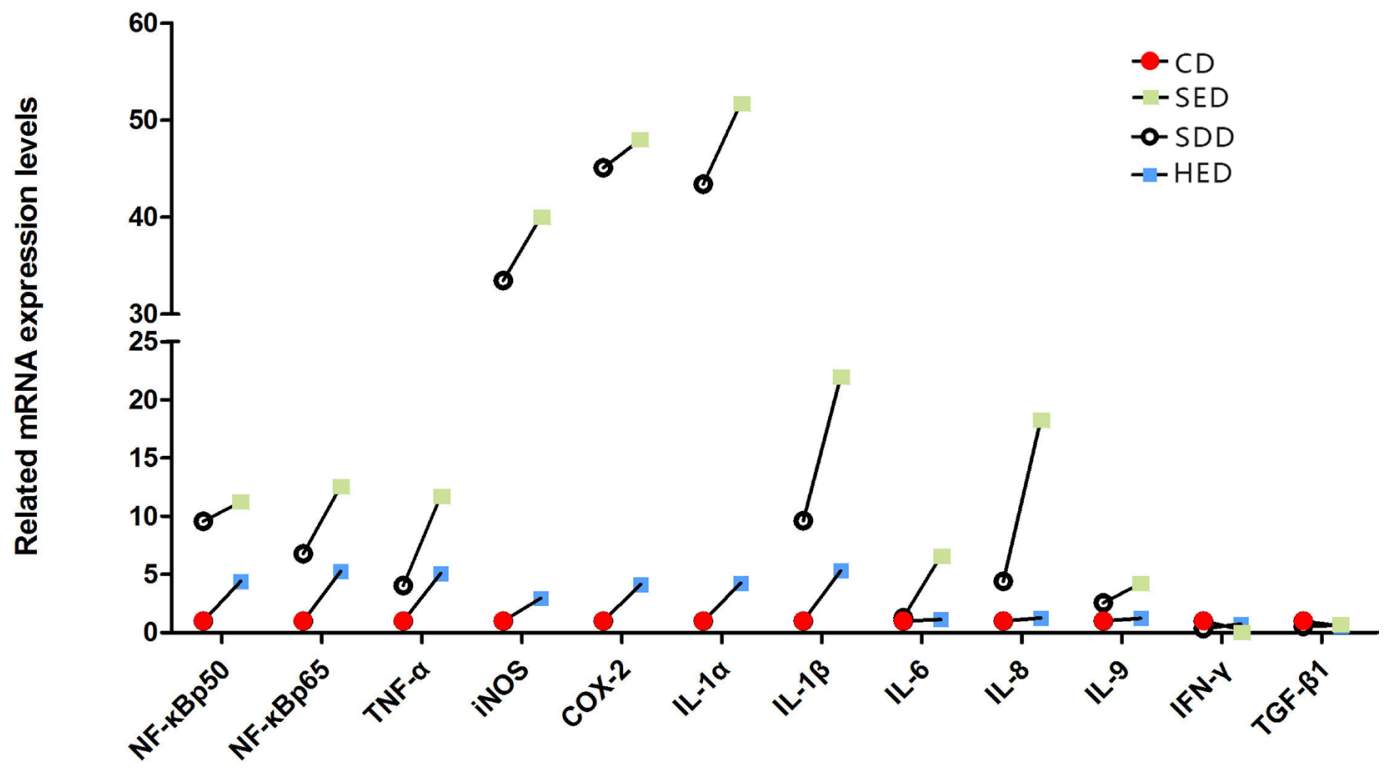

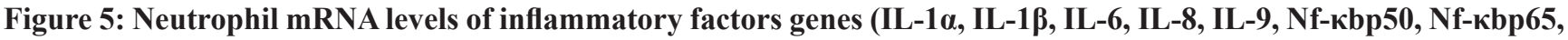
TNF- $\alpha$, iNOS, COX-2, IFN- $\boldsymbol{\gamma}$ and TGF- $\boldsymbol{\beta} 1)$ in different treated groups. Bars that do not share the same letters are significantly different $(p<0.05)$ from each other. The data were expressed as the mean $\pm \operatorname{SD}(n=10)$. 
would decrease the content of selenoproteins [46-48]. It is noteworthy that the expression of TRXR-1 reduced significantly in the $\mathrm{Se}^{-}$, energy ${ }^{+}$, and $\mathrm{Se}^{-}$-energy ${ }^{+}$ group and that the reduction peaked in the first group, implying that TRXR-1 probably participates in the Se-deficient inhibition of the Nrf2-pathway. On the one hand, the inhibited Nrf2 pathway induced oxidative stress in neutrophils. On the other hand, the decreased expression of selenoproteins provoked oxidative stress in neutrophils.

As the neutrophils act as an important defender of the peripheral immune system, its intracellular oxidative stress is closely related to phagocytosis. When phagocytosis occurs, oxidative burst occurs inside cells, along with the emergence of a vast number of ROS [49], propelling the Nrf2 pathway to produce antioxidants to down regulate oxidative burst and maintain the dynamic redox homeostasis [50]. While observing obese individuals, we found that macrophages infiltrated the adipose tissue and their phagocytosis deteriorated [51]. A low-selenium diet would exert similar impacts on neutrophils, as was demonstrated by Hogan JS, who studied the relationship between the Se content in cow's fodder and function of neutrophils in the 1990s, concluding that deficiency of Se could impair the phagocytic ability of neutrophils [52]. In this study, the Se-deficient, high-energy diet inhibited the activation of the Nrf2 pathway in neutrophils and the reduction of accumulated ROS, keeping neutrophils in a constant state of oxidative stress. Therefore, dysfunction of neutrophil phagocytosis might be impaired by the threshold of free oxygen radicals.

Neutrophils activated by oxidative stress generated a substantial amount of pro-inflammatory cytokines, including IL-6, IL-8, COX-2, and TNF- $\alpha$, and undermined the expression of anti-inflammatory factors, such as IFN- $\gamma$ and TGF- $\beta 1$ [20]. This was in line with what happened to neutrophils in our study. Studies on small-molecule organo-selenium found that its levels were related with chronic low-grade inflammation [53]. Studies on poultry and mice fed low Se diets also revealed that a low content of Se could induce inflammation in some organs and tissue $[54,55]$. Apart from that, obesity caused by high-fat would occasionally cause chronic low-grade inflammation characterized by macrophage infiltration in the adipose tissue where changes in relevant inflammatory factors, such as TNF- $\alpha$ and IL-1, were also detected [56]. Different behaviors of serum cytokines in the $\mathrm{Se}^{-}$, energy ${ }^{+}$, and $\mathrm{Se}^{-}$-energy ${ }^{+}$group did indicate that both a low level of Se and a high content of energy might result in chronic inflammation that would subject neutrophils to continuous exposure to oxidant and pro-inflammatory factors $[57,58]$. In this way, oxidative stress in neutrophils would continue and the accumulated ROS would do harm to cell structures [59], bringing extra damage to neutrophil phagocytosis.

In conclusion, through the histological investigation of neutrophil function against oxidative stress, this study has shown that the activation of the Nrf2 pathway in neutrophils can be inhibited by Se-deficient, high-energy diet. This further damages phagocytosis via chronic oxidative stress, as well as causing chronic inflammation in the body (Figure 6). Specifically, how Se deficiency and high-energy diet are involved in the Nrf2 pathway is not clear. We demonstrated that persistent oxidative stress is the key to impaired phagocytosis. The mechanistic basis of this difference remains to be explored.

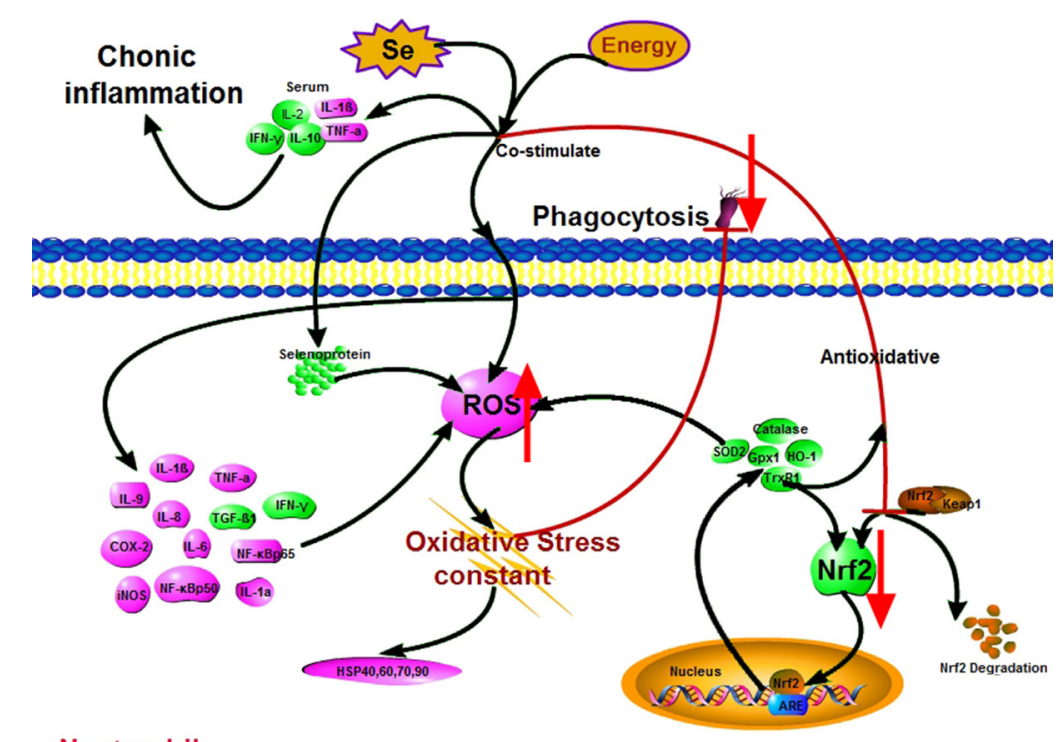

Neutrophil

Figure 6: Scheme shows mechanism of Nrf2 pathway-oxidative stress modulation due to $\mathrm{Se}^{-} / \mathrm{energy}^{+}$treatment. Pink denotes up-regulation (neutrophils: ROS, HSPs, IL-1 $\alpha$, IL-1 $\beta$, IL-6, IL-8, IL-9, TNF- $\alpha$, COX-2, iNOS, Nf-kbp50 and Nf-kbp65; serum: IL-1 $\beta$ and TNF- $\alpha$ ); green denotes down-regulation (neutrophils: Nrf2, SOD2, HO-1, catalase, Selenoproteins, IFN- $\gamma$ and TGF- $\beta 1$; serum: IL-2, IL-10 and IFN- $\gamma$ ). 


\section{MATERIALS AND METHODS}

\section{Treatment of experimental animals}

All procedures used in our study were approved by the Institutional Animal Care and Use Committee of Northeast Agricultural University. A $2 \times 2$ design trial was conducted that involved two dietary energy levels (normal DE v. high DE) and two dietary Se supplementation levels $(0$ and $0.3 \mathrm{mg} / \mathrm{kg}$ $\mathrm{Se}$ in the diet, the basal diet contains less than $0.03 \mathrm{mg} / \mathrm{kg}$ Se by analysis) (Supplementary Table 1). A total of 40 crossbred (Duroc x Landrace x Yorkshire, uncastrated) swine, with an average $\mathrm{BW}$ of $10 \pm 0.72 \mathrm{~kg}$, were randomly allotted to five groups $(n=10)$. All groups were fed with a Corn-Soybean meal diet, which was divided into three stages according to the growth stage of swine, stage I diet (10 30 kg BW), stage II diet (30 60 kg BW) and stage III diet $(60 \sim 110 \mathrm{~kg} \mathrm{BW})$. Some swine were maintained either on a Se-deficient diet ( $\mathrm{Se}^{-}$group, $\left.\mathrm{SDD}\right)$ with the basal diet, or on a sodium selenite diet (control group, CD). The other swine were maintained either on a high-energy diet (energy ${ }^{+}$group, HED) containing fat energy/digestion from $16 \% \sim 30 \%$, or on a Se-deficient, high-energy diet $\left(\mathrm{Se}^{-}\right.$-energy ${ }^{+}$group, $\left.\mathrm{SED}\right)$ supplemented with $0 \mathrm{mg} / \mathrm{kg}$ Se and fat energy/digestion $16 \% \sim 30 \%$. The basal diet contains $3296 \mathrm{~kJ} / \mathrm{kg}$ DE (stage I diet), $3294 \mathrm{~kJ} / \mathrm{kg}$ DE (stage II diet), and $3303 \mathrm{~kJ} / \mathrm{kg}$ DE (stage III diet). The high-energy diet contains $3492 \mathrm{~kJ} / \mathrm{kg} \mathrm{DE}$ (stage I diet), $3688 \mathrm{~kJ} / \mathrm{kg}$ DE (stage II diet), and $3929 \mathrm{~kJ} / \mathrm{kg}$ DE (stage III diet). Swine were euthanized after 16 weeks in the experiment, and the peripheral blood neutrophils were extracted for inspection. The neutrophils were frozen in liquid nitrogen with Trizol immediately and stored at $-80^{\circ} \mathrm{C}$ until required.

\section{Preparation of swine peripheral blood neutrophils suspension}

Fifteen milliliters of fresh porcine vein blood was mixed with sodium citrate tribasic in a ratio of seven to one and mixed into $15 \mathrm{ml}$ of PBS. The mix was added to the $15 \mathrm{ml}$ of neutrophil separation medium and centrifuged at $500 \mathrm{xg}$ for $20 \mathrm{~min}$. The second and third layer were collected into a tube containing $15 \mathrm{ml}$ of PBS, mixed well, and centrifuged at $250 \mathrm{xg}$ for $10 \mathrm{~min}$. After centrifuging, the supernatant was discarded and cells at the bottom of the tube were collected. The cells were mixed with $10 \mathrm{ml}$ of PBS. The mixed liquid was added into the lymphocyte separation medium and centrifuged at $500 \mathrm{xg}$ for $20 \mathrm{~min}$. Cells were recovered from the tube and were washed and re-suspended in PBS. Swine peripheral blood neutrophils were collected with $1 \mathrm{ml}$ of Trizol and stored immediately at $-80^{\circ} \mathrm{C}$ for the RNA isolation.

\section{Assessment of phagocytosis by neutrophils}

Phagocytic activity of swine neutrophils was measured with hydrophilic MSHP by a direct microscopic counting procedure, using a modified method described by Vetvicka et al., [60]. Blood smears were prepared and stained in accordance with methods explained by MayGrünwald and Giemsa-Romanowski [61]. One hundred neutrophils were examined to observe the phagocytic activity of neutrophils containing at least 3 engulfed particles in each smear. The phagocytic activity index was calculated as engulfed particles per total number of phagocytes observed. Increased intracellular fluorescence corresponds to increased phagocytosis.

\section{Measurement of ROS}

The concentration of separated neutrophils was adjusted to $106 / \mathrm{ml}$ by centrifuging $1 \mathrm{ml}$ of cell suspension at $500 \mathrm{xg}$ for $5 \mathrm{~min}$. After centrifuging, the supernatant was discarded, cells at the bottom of the tube were collected, and then the neutrophils were washed with PBS twice. After washing, $1 \mu$ of DCFH-DA fluorescent probe was added in the mix of neutrophils, incubated at $37^{\circ} \mathrm{C}$ for $30 \mathrm{~min}$, and washed with PBS. Then, $0.1 \mathrm{ml}$ of cell suspension was smeared, captured under a fluorescence microscope, and ROS-associated fluorescent signals were quantified with Image J software (Broken Symmetry Software, Albert, Cardona) [62]. The relative intensities were reported in arbitrary units per cell.

\section{Western blot analysis}

Total protein was assessed with SDS-polyacrylamide gel electrophoresis under reducing conditions on $12 \%$ gels and then transferred to nitrocellulose membranes using a tank transfer at $80 \mathrm{mV}$ in Tris-glycine buffer containing $20 \%$ methanol for $2 \mathrm{~h}$. Nitrocellulose membranes were blocked for $2 \mathrm{~h}$ with $5 \%$ skim milk at $37^{\circ} \mathrm{C}$ and incubated for $12 \mathrm{~h}$ with diluted primary swine antibody $\mathrm{Nrf} 2$ (1:1000), SOD2 (1:1000), HO-1 (1:1000), Gpx1 (1:1000), TrxR1 $(1: 1000)$ and Catalase $(1: 1000)$ at $4^{\circ} \mathrm{C}$. This was then followed incubated with a horseradish peroxidaseconjugated secondary antibody against rabbit $\operatorname{IgG}(1: 1500$, Santa Cruz, CA, USA) using the ECL kit (Kangweishiji Biotechnology, Beijing, China). For statistical analysis, a box plot analysis was applied.

\section{ELISA based measurement of cytokines activity}

The concentration of serum cytokines (IL-1 $\beta$, IL-2, IL-10, TNF- $\alpha$, IFN- $\gamma$ ) was determined using an ELISA kit (Hermes Criterion Biotechnology, Canada). The level of substance was detected in the sample kit using a double antibody sandwich method. Microtiter plate wells were coated with monoclonal antibody. The test substance was then added, and combined with the HRP-labeled test substance antibody, becoming antibody-antigen-enzyme labeled antibody complexes. After washing completely, TMB substrate was added. HRP-catalyzed reactions are blue, and turn yellow as the reaction progresses. The color 
in a sample substance was positively correlated with the amount of substance. We determined the concentration of a substance in the sample by comparing the absorbance at $450 \mathrm{~nm}$ wavelength, calculated by a standard curve.

\section{Quantitative real time-polymerase chain reaction}

Total RNA was isolated from neutrophils using Trizol reagent according to the manufacturer's instructions (Invitrogen, Carlsbad, USA). The cDNA was synthesized using the Revert Aid First Strand cDNA Synthesis Kit (Roche, Basel, Switzerland). The cDNA was detected by Quantitative Real Time PCR (qRT-PCR). The specific primers (Supplementary Table 2) of selenoproteins, HSPs, inflammatory factors, GAPDH and $\beta$-actin based on known swine sequences were designed by Primer Premier Software (PREMIER Biosoft International, USA). $\beta$-actin was used as an internal reference. qRT-PCR was performed on a Light Cycler ${ }^{\circledR} 480$ System (Roche, Basel, Switzerland) using Fast Universal SYBR Green Master (Roche, Basel, Switzerland). Only one peak for each PCR product was shown in the melting curve analysis. The relative abundance of mRNA was calculated according to the method of $2^{-\Delta \Delta \mathrm{Ct}}$ [63], accounting for gene-specific efficiencies and was normalized to the mean expression of the above-mentioned index.

\section{Statistical analysis}

Statistical analyses of all data were performed with GraphPad Prism (version 5.0, Graph Pad Software Inc., San Diego, CA, USA). All data were analyzed by one-way ANOVA analysis, had a normal distribution and passed equal variance testing. Quantitative data are presented as the mean \pm SD. Samples with different superscript letters represented statistically significant differences $(P<0.05)$.

\section{Abbreviations}

Nrf2, nuclear factor E2-related factor 2; ROS, reactive oxygen species; HSPs, heat shock proteins; Gpx, glutathione peroxidase; TrxR, thioredoxin reductase; Sepp1, selenoprotein P; Seln, selenoprotein N; Selw, selenoprotein W; IL-1, interlenkin-1 $\beta$; IL-6, interlenkin-6; IL-10, interlenkin-10; TNF- $\alpha$, tumor necrosis factor $\alpha$; COX-2, cyclooxygenaase; Keap1, Kelch like-ECHassociated protein 1; ARE, antioxidant-response element; SOD2, superoxide dismutase 2; HO-1, haemoxygenase-1; ApoE, apolipoprotein E; MCP-1, monocyte chemotactic protein 1; DE, digestive energy; BW, body weight ; MSHP, microspheric particles.

\section{ACKNOWLEDGMENTS}

The authors thank Prof. Shiwen Xu for providing the instruments, and also thank Ms. Jing Fu for the careful language correction.

\section{CONFLICTS OF INTEREST}

No, there is no conflicts of interest that I should disclose, having read the above statement.

\section{FUNDING}

This work was supported by the National Science and Technology Support Program (2013BAD20B04), China Agriculture Research System(CARS-36).

\section{REFERENCES}

1. Hoffmann PR, Berry MJ. The influence of selenium on immune responses. Molecular nutrition \& food research. 2008; 52:1273-1280.

2. Nijhuis J, Rensen SS, Slaats Y, van Dielen FM, Buurman WA, Greve JW. Neutrophil activation in morbid obesity, chronic activation of acute inflammation. Obesity. 2009; 17:2014-2018

3. Tateya S, Kim F, Tamori Y. Recent advances in obesityinduced inflammation and insulin resistance. Frontiers in endocrinology. 2013; 4:93.

4. Khoso PA, Yang Z, Liu C, Li S. Selenium Deficiency Downregulates Selenoproteins and Suppresses Immune Function in Chicken Thymus. Biological trace element research. 2015; 167:48-55.

5. Liang Y, Lin SL, Wang CW, Yao HD, Zhang ZW, Xu SW. Effect of selenium on selenoprotein expression in the adipose tissue of chickens. Biological trace element research. 2014; 160:41-48.

6. Yao H, Zhao W, Zhao X, Fan R, Khoso PA, Zhang Z, Liu W, $\mathrm{Xu}$ S. Selenium deficiency mainly influences the gene expressions of antioxidative selenoproteins in chicken muscles. Biological trace element research. 2014; 161:318-327.

7. Strandberg L, Verdrengh M, Enge M, Andersson N, Amu S, Onnheim K, Benrick A, Brisslert M, Bylund J, Bokarewa M, Nilsson S, Jansson JO. Mice chronically fed high-fat diet have increased mortality and disturbed immune response in sepsis. PloS one. 2009; 4:e7605.

8. van der Heijden RA, Bijzet J, Meijers WC, Yakala GK, Kleemann R, Nguyen TQ, de Boer RA, Schalkwijk CG, Hazenberg BP, Tietge UJ, Heeringa P. Obesity-induced chronic inflammation in high fat diet challenged C57BL/6J mice is associated with acceleration of age-dependent renal amyloidosis. Scientific reports. 2015; 5:16474.

9. Stewart JD, Hengstler JG, Bolt HM. Control of oxidative stress by the Keap1-Nrf2 pathway. Archives of toxicology. $2011 ; 85: 239$.

10. Kansanen E, Kuosmanen SM, Leinonen H, Levonen AL. The Keap1-Nrf2 pathway: Mechanisms of activation and dysregulation in cancer. Redox biology. 2013; 1:45-49.

11. Araujo VC, Demasi AP, Soares AB, Passador-Santos F, Napimoga MH, Martinez EF, Freitas NS, Araujo NS. 
Neutrophils in oral paracoccidioidomycosis and the involvement of Nrf2. PloS one. 2013; 8:e76976.

12. Moon SY, Lee JH, Choi HY, Cho IJ, Kim SC, Kim YW. Tryptanthrin protects hepatocytes against oxidative stress via activation of the extracellular signal-regulated kinase/NF-E2-related factor 2 pathway. Biological \& pharmaceutical bulletin. 2014; 37:1633-1640.

13. Han XAY, Hamdam JM, Alhuseini LMA, Sethu S, Djouhri L, Walsh J, Kitteringham N, Park BK, Goldring CE, Sathish JG. Loss of Transcription Factor Nuclear FactorErythroid 2 (NF-E2) p45-related Factor-2 (Nrf2) Leads to Dysregulation of Immune Functions, Redox Homeostasis, and Intracellular Signaling in Dendritic Cells. Journal of Biological Chemistry. 2012; 287:10556-10564.

14. Stefanson AL, Bakovic M. Dietary regulation of Keap1/ Nrf2/ARE pathway: focus on plant-derived compounds and trace minerals. Nutrients. 2014; 6:3777-3801.

15. Reszka E, Wieczorek E, Jablonska E, Janasik B, Fendler W, Wasowicz W. Association between plasma selenium level and NRF2 target genes expression in humans. Journal of trace elements in medicine and biology : organ of the Society for Minerals and Trace Elements. 2015; 30:102-106.

16. Wang X, Li C, Xu S, Ishfaq M, Zhang X. NF-E2-related factor 2 deletion facilitates hepatic fatty acids metabolism disorder induced by high-fat diet via regulating related genes in mice. Food and chemical toxicology. 2016; 94:186-196.

17. Lopes RA, Neves KB, Tostes RC, Montezano AC, Touyz RM. Downregulation of Nuclear Factor Erythroid 2-Related Factor and Associated Antioxidant Genes Contributes to Redox-Sensitive Vascular Dysfunction in Hypertension. Hypertension. 2015; 66:1240-1250.

18. Kubben N, Zhang W, Wang L, Voss TC, Yang J, Qu J, Liu GH, Misteli T. Repression of the Antioxidant NRF2 Pathway in Premature Aging. Cell. 2016; 165:1361-1374.

19. Cao Y, Li X, Wang CJ, Li P, Yang B, Wang CB, Wang LX. Role of NF-E2-related factor 2 in neuroprotective effect of 1-carnitine against high glucose-induced oxidative stress in the retinal ganglion cells. Biomedicine \& pharmacotherapy = Biomedecine \& pharmacotherapie. 2015; 69:345-348.

20. Luo HR, Mondal S. Molecular control of PtdIns $(3,4,5)$ P3 signaling in neutrophils. EMBO reports. 2015; 16:149-163.

21. Petropoulos M, Karamolegkou G, Rosmaraki E, Tsakas S. Hydrogen peroxide signals E. coli phagocytosis by human polymorphonuclear cells; up-stream and down-stream pathway. Redox biology. 2015; 6:100-105.

22. Sima C, Aboodi GM, Lakschevitz FS, Sun C, Goldberg MB, Glogauer M. Nuclear Factor Erythroid 2-Related Factor 2 Down-Regulation in Oral Neutrophils Is Associated with Periodontal Oxidative Damage and Severe Chronic Periodontitis. The American journal of pathology. 2016; 186:1417-1426.

23. Del TR, Chèvre $R$, Rodríguez $\mathrm{C}$, Ordóñez $\mathrm{A}$, Martínezgonzález J, Andrés V, Méndezferrer S. Nestin(+) cells direct inflammatory cell migration in atherosclerosis. $2016 ; 7$.
24. Osaka M, Ito $\mathrm{S}$, Honda $\mathrm{M}$, Inomata $\mathrm{Y}$, Egashira $\mathrm{K}$, Yoshida M. Critical role of the C5a-activated neutrophils in high-fat diet-induced vascular inflammation. Scientific reports. 2016; 6 .

25. Lescure A, Rederstorff M, Krol A, Guicheney P, Allamand V. Selenoprotein function and muscle disease. Biochimica et biophysica acta. 2009; 1790:1569-1574.

26. Gao X, Zhang Z, Li Y, Shen P, Hu X, Cao Y, Zhang N. Selenium Deficiency Facilitates Inflammation Following S. aureus Infection by Regulating TLR2-Related Pathways in the Mouse Mammary Gland. Biological trace element research. 2016; 172:449-457.

27. Carlson BA, Yoo MH, Shrimali RK, Irons R, Gladyshev VN, Hatfield DL, Park JM. Role of seleniumcontaining proteins in T-cell and macrophage function. The Proceedings of the Nutrition Society. 2010; 69:300-310.

28. Furukawa S, Fujita T, Shimabukuro M, Iwaki M, Yamada Y, Nakajima Y, Nakayama O, Makishima M, Matsuda M, Shimomura I. Increased oxidative stress in obesity and its impact on metabolic syndrome. The Journal of clinical investigation. 2004; 114:1752-1761.

29. Lecube A, Pachon G, Petriz J, Hernandez C, Simo R. Phagocytic activity is impaired in type 2 diabetes mellitus and increases after metabolic improvement. PloS one. 2011; 6:e23366.

30. Khoso PA, Yang Z, Liu C, Li S. Selenium Deficiency Downregulates Selenoproteins and Suppresses Immune Function in Chicken Thymus. Biological trace element research. 2015; 167:1-8.

31. Zhang ZW, Wang QH, Zhang JL, Li S, Wang XL, Xu SW. Effects of oxidative stress on immunosuppression induced by selenium deficiency in chickens. Biological trace element research. 2012; 149:352-361.

32. Kioussi C, Whanger PD. Selenoprotein W in development and oxidative stress: Springer US). 2006.

33. Labunskyy VM, Hatfield DL, Gladyshev VN. Selenoproteins: Molecular Pathways and Physiological Roles. Physiological reviews. 2014; 94:739-777.

34. Chen X, Yao H, Yao L, Zhao J, Luan Y, Zhang Z, Xu S. Selenium deficiency influences the gene expressions of heat shock proteins and nitric oxide levels in neutrophils of broilers. Biological trace element research. 2014; 161:334-340.

35. Zhao H, Li K, Tang JY, Zhou JC, Wang KN, Xia XJ, Lei XG. Expression of Selenoprotein Genes Is Affected by Obesity of Pigs Fed a High-Fat Diet. The Journal of nutrition. 2015; 145:1394-1401.

36. Kaya A, Uzunhasan I, Baskurt M, Ozkan A, Ataoglu E, Okcun B, Yigit Z. Oxidative status and lipid profile in metabolic syndrome: gender differences. Metabolic syndrome and related disorders. 2010; 8:53-58.

37. Xu H, Barnes GT, Yang Q, Tan G, Yang D, Chou CJ, Sole J, Nichols A, Ross JS, Tartaglia LA, Chen H. Chronic inflammation in fat plays a crucial role in the development of obesity-related insulin resistance. The Journal of clinical investigation. 2003; 112:1821-1830. 
38. Griendling KK, Sorescu D, Ushio-Fukai M. NAD(P) $\mathrm{H}$ oxidase: role in cardiovascular biology and disease. Circulation research. 2000; 86:494-501.

39. Zhang X, Dong F, Ren J, Driscoll MJ, Culver B. High dietary fat induces NADPH oxidase-associated oxidative stress and inflammation in rat cerebral cortex. Experimental neurology. 2005; 191:318-325.

40. Macedo LF, Rogero MM, Guimaraes JP, Granato D, Lobato LP, Castro IA. Effect of red wines with different in vitro antioxidant activity on oxidative stress of high-fat diet rats. Food chemistry. 2013; 137:122-129.

41. Oprescu AI, Bikopoulos G, Naassan A, Allister EM, Tang C, Park E, Uchino H, Lewis GF, Fantus IG, RozakisAdcock M, Wheeler MB, Giacca A. Free fatty acid-induced reduction in glucose-stimulated insulin secretion: evidence for a role of oxidative stress in vitro and in vivo. Diabetes. 2007; 56:2927-2937.

42. Lin H, Lu JP, Laflamme P, Qiao S, Shayegan B, Bryskin I, Monardo L, Wilson BC, Singh G, Pinthus JH. Inter-related in vitro effects of androgens, fatty acids and oxidative stress in prostate cancer: a mechanistic model supporting prevention strategies. International journal of oncology. 2010; 37:761-766.

43. Song Y, Li X, Li Y, Li N, Shi X, Ding H, Zhang Y, Li X, Liu G, Wang Z. Non-esterified fatty acids activate the ROS-p38-p53/Nrf2 signaling pathway to induce bovine hepatocyte apoptosis in vitro. Apoptosis : an international journal on programmed cell death. 2014; 19:984-997.

44. Morrison CD, Pistell PJ, Ingram DK, Johnson WD, Liu Y, Fernandez-Kim SO, White CL, Purpera MN, Uranga RM, Bruce-Keller AJ, Keller JN. High fat diet increases hippocampal oxidative stress and cognitive impairment in aged mice: implications for decreased $\mathrm{Nrf} 2$ signaling. Journal of neurochemistry. 2010; 114:1581-1589.

45. Muller M, Banning A, Brigelius-Flohe R, Kipp A. Nrf2 target genes are induced under marginal seleniumdeficiency. Genes \& nutrition. 2010; 5:297-307.

46. Huang JQ, Li DL, Zhao H, Sun LH, Xia XJ, Wang KN, Luo X, Lei XG. The selenium deficiency disease exudative diathesis in chicks is associated with downregulation of seven common selenoprotein genes in liver and muscle. Journal of Nutrition. 2011; 141:1605-1610.

47. Liu CP, Fu J, Lin SL, Wang XS, Li S. Effects of Dietary Selenium Deficiency on mRNA Levels of Twenty-One Selenoprotein Genes in the Liver of Layer Chicken. Biological trace element research. 2014; 159:192-198.

48. Tsuji PA, Carlson BA, Anderson CB, Seifried HE, Hatfield DL, Howard MT. Dietary Selenium Levels Affect Selenoprotein Expression and Support the Interferon- $\gamma$ and IL-6 Immune Response Pathways in Mice. Nutrients. 2015; 7:6529-6549.

49. Kang YP, Zhou Y, Xue XY. [Synergistic anti-inflammatory effects in the process of neutriphil phagocytosis by macrophage]. Sheng li ke xue jin zhan [Progress in physiology]. 2012; 43:455-458.

50. Chacko BK, Wall SB, Kramer PA, Ravi S, Mitchell T, Johnson MS, Wilson L, Barnes S, Landar A, DarleyUsmar VM. Pleiotropic effects of 4-hydroxynonenal on oxidative burst and phagocytosis in neutrophils. Redox biology. 2016; 9:57-66.

51. Dam ADV. Impaired phagocytosis as a key mechanism underlying obesity-induced inflammation and insulin resistance. Front Endocrinol. 2014; 5:204.

52. Hogan JS, Smith KL, Weiss WP, Todhunter DA, Schockey WL. Relationships Among Vitamin E, Selenium, and Bovine Blood Neutrophils 1. Journal of dairy science. 1990; 73:2372-2378.

53. Zhou J, Xu H, Huang K. Organoselenium Small Molecules and Chromium(III) Complexes for Intervention in Chronic Low-grade Inflammation and Type 2 Diabetes. Current topics in medicinal chemistry. 2016; 16:823-834.

54. Felix K, Gerstmeier S, Kyriakopoulos A, Howard OM, Dong HF, Eckhaus M, Behne D, Bornkamm GW, Janz S. Selenium deficiency abrogates inflammation-dependent plasma cell tumors in mice. Cancer research. 2004; 64:2910-2917.

55. Wei Z, Yao M, Li Y, He X, Yang Z. Dietary selenium deficiency exacerbates lipopolysaccharide-induced inflammatory response in mouse mastitis models. Inflammation. 2014; 37:1925-1931.

56. Maury E, Brichard SM. Adipokine dysregulation, adipose tissue inflammation and metabolic syndrome. Molecular and cellular endocrinology. 2010; 314:1-16.

57. Mera K, Anraku M, Kitamura K, Nakajou K, Maruyama T, Otagiri M. The structure and function of oxidized albumin in hemodialysis patients: Its role in elevated oxidative stress via neutrophil burst. Biochemical \& Biophysical Research Communications. 2005; 334:1322-1328.

58. Kolli VK, Abraham P, Isaac B, Selvakumar D. Neutrophil infiltration and oxidative stress may play a critical role in methotrexate-induced renal damage. Chemotherapy. 2009; 55:83-90.

59. $\dagger$ FAL, $\dagger$ A-IK, Heinrich L, Birck R, Kallenberg CGM, Heeringa P, Yard B. Inhibition of neutrophil-mediated production of reactive oxygen species (ROS) by endothelial cells is not impaired in anti-neutrophil cytoplasmic autoantibodies (ANCA)-associated vasculitis patients. Clinical \& Experimental Immunology. 2010; 161:268-275.

60. Větvička V, Fornůsek L, Kopeček J, Kamínková J, Kašpárek L, Vránová M. Phagocytosis of human blood leukocytes: A simple micromethod. Immunology letters. 1982; 5:97-100.

61. K Č, Faix Š, Plachá I, Mihaliková K, Váradyová Z, Kišidayová S, Grešáková L. Effects of Different Dietary Selenium Sources on Antioxidant Status and Blood Phagocytic Activity in Sheep. Biological trace element research. 2016:1-8. 
62. Liu X, Ward K, Xavier C, Jann J, Clark AF, Pang IH, Wu H. The novel triterpenoid RTA 408 protects human retinal pigment epithelial cells against $\mathrm{H} 2 \mathrm{O} 2$-induced cell injury via NF-E2-related factor 2 (Nrf2) activation. Redox biology. 2016; 8:98-109.
63. Livak KJ, Schmittgen TD. Analysis of Relative Gene Expression Data Using Real-Time Quantitative PCR and the $2^{-\triangle \Delta \mathrm{CT}}$ Method. Methods. 2001; 25:402-408. 